J. Austral. Math. Soc. (Series A) 28 (1979), 129-135

\title{
EFFECTIVE BOUNDS OF THE SOLUTIONS OF CERTAIN DIOPHANTINE EQUATIONS
}

\author{
N. I. FELDMAN
}

\section{Dedicated to K. Mahler for his 75th birthday}

(Received 22 November 1978)

Communicated by J. H. Coates

\begin{abstract}
The paper establishes an effective bound for the solutions of the equation$$
\operatorname{Norm}_{K / Q}\left(x_{1} \alpha_{1}+x_{2} \alpha_{2}+x_{3} \alpha_{3}\right)=a, a \in Z,
$$

under some conditions.

Subject classification (Amer. Math. Sac. (MOS) 1970): 10 B 15.
\end{abstract}

1.

Let $\mathbf{K}$ be a field of algebraic numbers of degree $p \geqslant 3, \alpha_{1}, \ldots, \alpha_{n}$-linearly independent over $\mathbf{Q}$ integers from $\mathbf{K}, a \in \mathbf{Z}$. It is known that if $m=p$, then the equation

$$
F\left(x_{1}, \ldots, x_{m}\right)=\operatorname{Norm}_{\mathrm{K} / \mathrm{Q}}\left(x_{1} \alpha_{1}+\ldots+x_{m} \alpha_{m}\right)=a
$$

for some $a$ (for example, $a=1$ ) has infinitely many solutions in rational integers $x_{1}, \ldots, x_{m}$. If $m<p$, then excepting the case when the modulus $\mathscr{M}\left(\alpha_{1}, \ldots, \alpha_{m}\right)$ is degenerate, this equation for any $a \in \mathbf{Z}$ may have only finitely many solutions. For $m=2$ it was proved in 1909 by Thue (1909) and for $m \geqslant 3$ in 1971 by Schmidt (1971). The theorems proved by Thue and Schmidt are noneffective-we cannot obtain from them an upper bound depending on $F$ and $a$ of the absolute values of

(C) Copyright Australian Mathematical Society 1979

Copyright. Apart from any fair dealing for scholarly purposes as permitted under the Copyright Act, no part of this JOURNAI may be reproduced by any process without written permission from the Treasurer of the Australian Mathematical Society. 
the numbers $x_{1}, \ldots, x_{m}$ satisfying the equation. In 1968 Baker (1968) made Thue's theorem effective. In this paper some effective results are obtained for $m=3$.

\section{2.}

Let the degree of $K$ be $2 n \geqslant 4$, the fields $K_{1}=K, K_{2}, \ldots, K_{2 n}$ be conjugate and complex, $\mathbf{K}_{s}$ and $\mathbf{K}_{s+n}$ be complex conjugate $\left(\mathbf{K}_{s}=\overline{\mathbf{K}}_{s+n}\right), s=1, \ldots, n$. Let " $\alpha=\alpha^{(1)}, \beta=\beta^{(1)}$ and $\gamma=\gamma^{(1)}$ be linearly independent over $\mathbf{Q}$ integers from $\mathbf{K}$, $\alpha^{(l)}, \beta^{(l)}, \gamma^{(i)}$-their conjugates in the field $\mathbf{K}^{(1)}$. Let us investigate the solutions in rational integers of the diophantine equations

$$
F(x, y, z)=\prod_{t=1}^{2 n}\left(x \alpha^{(t)}+y \beta^{(t)}+z \gamma^{(t)}\right)=a, \quad a \in \mathbf{Z} .
$$

Let

$$
\begin{array}{cl}
\delta_{i 1}=\beta^{(i)} \bar{\gamma}^{(i)}+\bar{\beta}^{(i)} \gamma^{(i)}, & \delta_{i 2}=\gamma^{(i)} \bar{\alpha}^{(i)}-\bar{\gamma}^{(i)} \alpha^{(i)}, \\
\delta_{i 3}=\alpha^{(i)} \bar{\beta}^{(i)}-\bar{\alpha}^{(i)} \beta^{(i)}, & i=1, \ldots, n, \\
\Delta_{i j 1}=\delta_{i 2} \delta_{j 3}-\delta_{i 3} \delta_{j 2}, & \Delta_{i j 2}=\delta_{i 3} \delta_{j 1}-\delta_{i 1} \delta_{j 3}, \\
\Delta_{i j 3}=\delta_{i 1} \delta_{j 2}-\delta_{i 2} \delta_{j 1}, & i=j, 1, \ldots, n .
\end{array}
$$

In what follows the letters $c, c_{0}, \ldots$ denote effective positive constants, depending only on $\alpha, \beta, \gamma$ and $\mathbf{K}$ but not on $a$.

THEOREM 1. If for all the pairs $i, j, i \neq j, 1<i, j<n$, the numbers $\Delta_{i j 1}, \Delta_{i j 2}, \Delta_{i j 3}$ are linearly independent over $\mathbf{Q}$, then for any $a \in \mathbf{Z}$ all the integer numbers $x, y, z$ satisfying the equation (1) also satisfy the inequality

$$
|x|,|y|,|z| \leqslant c_{1}|a| c_{3} .
$$

PROOF. Let $x, y, z$ be the solution of the equation (1). If $a=0$ then $\alpha x+\beta y+\gamma z=0$ and the linear independence of $\alpha, \beta$ and $\gamma$ yields that $x=y=z=0$, that is, that (4) is true. In what follows we consider $a \neq 0$. Let

$$
\lambda^{(l)}=x \alpha^{(i)}+y \beta^{(i)}+z \gamma^{(i)}, \quad \prod_{i=1}^{2 n} \lambda^{(i)}=a,
$$

$\eta_{1}, \ldots, \eta_{r}$ be the fundamental units of the field $\mathbf{K}$, and the numbers $\eta_{1}^{(i)}, \ldots, \eta_{r}^{(t)}$ their conjugates in $\mathbf{K}^{(t)}$. In the paper Baker $(1968)$ it is proved that there exist such $b_{1}, \ldots, b_{r} \in \mathbf{Z}$ for which

$$
\begin{aligned}
c_{3}^{-1}|a|^{1 / 2 n} \leqslant\left|\mu^{(t)}\right| \leqslant c_{3}|a|^{1 / 2 n}, \quad \mu^{(t)}=\lambda^{(t)} \eta_{1}^{(t)_{1}^{b_{1}}} \ldots \eta_{r}^{()^{b_{r}}=}=\lambda^{(t)} \zeta^{(t)}, \\
t=1,2, \ldots, 2 n,
\end{aligned}
$$


and if $H=\max \left|b_{l}\right|$ then there exist such indices $k$ and $l$ that

$$
\left|\lambda^{(k)}\right| \leqslant|a|^{1 / 2 n} e^{-c_{4} H}, \quad\left|\lambda^{(l)}\right| \geqslant c_{5}|a|^{1 / 2 n} \text {. }
$$

It is evident that the linear forms $\bar{\lambda}^{(k)}$ and $\bar{\lambda}^{(l)}$-complex conjugate to $\lambda^{(k)}$ and $\lambda^{(l)}$ satisfy just the same inequalities. The system of equations

$$
\begin{aligned}
\alpha^{(k)} u+\beta^{(k)} v+\gamma^{(k)} w+\lambda^{(k)} t & =0, \\
\bar{\alpha}^{(k)} u+\bar{\beta}^{(k)} v+\bar{\gamma}^{(k)} w+\bar{\lambda}^{(k)} t & =0, \\
\alpha^{(l)} u+\beta^{(l)} v+\gamma^{(l)} w+\lambda^{(l)} t & =0, \\
\bar{\alpha}^{(l)} u+\bar{\beta}^{(l)} v+\bar{\gamma}^{(l)} w+\bar{\lambda}^{(l)} t & =0
\end{aligned}
$$

has a nontrivial solution $(x, y, z,-1)$ then the determinant is equal to zero, that is,

$$
D_{1} \lambda^{(k)}+\bar{D}_{1} \bar{\lambda}^{(k)}+D_{2} \lambda^{(l)}+\bar{D}_{2} \bar{\lambda}^{(l)}=0,
$$

where

$$
D_{1}=\left|\begin{array}{ccc}
\bar{\alpha}^{(k)} & \bar{\beta}^{(k)} & \bar{\gamma}^{(k)} \\
\alpha^{(l)} & \beta^{(l)} & \gamma^{(l)} \\
\bar{\alpha}^{(l)} & \bar{\beta}^{(l)} & \bar{\gamma}^{(l)}
\end{array}\right|, \quad D_{2}=\left|\begin{array}{ccc}
\alpha^{(k)} & \beta^{(k)} & \gamma^{(k)} \\
\bar{\alpha}^{(k)} & \bar{\beta}^{(k)} & \bar{\gamma}^{(k)} \\
\bar{\alpha}^{(l)} & \bar{\beta}^{(l)} & \bar{\gamma}^{(l)}
\end{array}\right|,
$$

and $\bar{D}_{1}, \bar{D}_{2}$ are their complex conjugates. From (7) and (8) we obtain

$$
\left|D_{2} \lambda^{(l)}+\bar{D}_{2} \bar{\lambda}^{(l)}\right|<c_{6}|a|^{1 / 2 n} e^{-c_{4} H} \text {. }
$$

If the equality

$$
D_{2} \lambda^{(l)}+\bar{D}_{2} \bar{\lambda}^{(l)}=0
$$

takes place then, taking into account (9), we would have

$$
\begin{aligned}
0 & =\left(x \alpha^{(l)}+y \beta^{(l)}+z \gamma^{(l)}\right) D_{2}+\left(x \bar{\alpha}^{(l)}+y \bar{\beta}^{(l)}+z \gamma^{(l)}\right) D_{2} \\
& =x\left|\begin{array}{ccc}
\alpha^{(k)} & \beta^{(k)} & \gamma^{(k)} \\
\bar{\alpha}^{(k)} & \bar{\beta}^{(k)} & \bar{\gamma}^{(k)} \\
0 & \delta_{l 3} & -\delta_{l 2}
\end{array}\right|+y\left|\begin{array}{ccc}
\alpha^{(k)} & \beta^{(k)} & \gamma^{(k)} \\
\bar{\alpha}^{(k)} & \bar{\beta}^{(k)} & \bar{\gamma}^{(k)} \\
-\delta_{l 3} & 0 & \delta_{l 1}
\end{array}\right|+z\left|\begin{array}{ccc}
\alpha^{(k)} & \beta^{(k)} & \gamma^{(k)} \\
\bar{\alpha}^{(k)} & \bar{\beta}^{(k)} & \bar{\gamma}^{(k)} \\
\delta_{l 2} & -\delta_{l 1} & 0
\end{array}\right| \\
& =x\left(\delta_{k 2} \delta_{l 3}-\delta_{k 3} \delta_{l 2}\right)+y\left(\delta_{k 3} \delta_{l 1}-\delta_{k 1} \delta_{l 3}\right)+z\left(\delta_{k 1} \delta_{l 2}-\delta_{k 2} \delta_{l 1}\right) \\
(11) & =x \Delta_{k l 1}-y \Delta_{k l 2}+z \Delta_{k l 3} .
\end{aligned}
$$

But by the conditions of the theorem this equality can take place only for $x=y=z=0$ but this triplet is not the solution of (1) as $a \neq 0$. Thus from (6), (10) and (11) we obtain

$$
0<\left|D_{2} \mu^{(l)} \zeta^{(l)^{-1}}+\bar{D}_{2} \bar{\mu}^{(l)} \bar{\zeta}^{(l)-1}\right|<c_{6}|a|^{1 / 2 n} e^{-c_{4} H} .
$$


This implies that $D_{2} \neq 0$ and therefore, on multiplying the inequality (12) by $\left|\zeta^{(l)} / D_{2} \mu^{(l)}\right|$, from (6), (7), (12) and taking into account that $D_{2}$ belongs to a fixed finite set of numbers we obtain the inequality

$$
0<\left|\frac{\bar{\zeta}^{(l)}}{\zeta^{(l)}}+\frac{\bar{D}_{2} \bar{\mu}^{(l)}}{D_{2} \mu^{(l)}}\right|<c_{6} e^{-c_{4} H}|a|^{1 / 2 n}\left|D_{2}^{-1} \bar{\zeta}^{(l)}\right|\left|\lambda^{(l)} \zeta^{(l)}\right|^{-1} c_{7} e^{-c_{6} H},
$$

or

$$
0<\left|\left(\frac{\bar{\eta}_{1}^{(l)}}{\eta_{1}^{(l)}}\right)^{b_{1}} \ldots\left(\frac{\bar{\eta}_{r}^{(l)}}{\zeta_{r}^{(l)}}\right)^{b_{2}}-\omega\right|<c_{7} e^{-c_{4} H}
$$

where

$$
\omega=-\frac{\bar{D}_{2}}{D_{2}} \frac{\bar{\mu}^{(l)}}{\mu^{(l)}}
$$

In the papers Фельдман (1971) and Фельдман (1972) the theorem is proved:

THEOREM $\dagger$. Let $\omega_{1}, \ldots, \omega_{m}, \omega$ be algebraic from the field $\mathbf{K}_{0}$ and different from zero, $b_{1}, \ldots, b_{m} \in \mathbf{Z}, H=\max \left|b_{k}\right|, \delta>0,|\omega|,\left|\omega^{-1}\right|<c$. If

then

$$
0<\left|\omega_{1}^{b_{1}} \ldots \omega_{m}^{b_{m}}-\omega\right|<e^{-\delta H},
$$

$$
H<c_{0}(1+\log H(\omega)), c_{0}=c_{0}\left(\omega_{1}, \ldots, \omega_{2}, \mathbf{K}, \delta, c\right) .
$$

Let us apply this theorem to the inequality (14) where $m=r, \omega_{j}=\bar{\eta}_{j}^{(l)} / \eta_{j}^{(l)}$ and $\omega$ is defined by (15). We can obtain all the conjugates of $\omega$ by replacing the numbers $D_{2}, \bar{D}_{2}, \mu^{(l)}, \bar{\mu}^{(l)}$ with some of their conjugates and according to (6) the numbers $|\omega|$ and $\left|\omega^{-1}\right|$ are bounded. Thus (16) holds. The heights of the numbers $D_{2}$ and $\bar{D}_{2}$ are bounded and the heights of the integers $\bar{\mu}^{(l)}$ and $\bar{\mu}^{(l)}$ are not greater than $c_{8}|a|$ because of (6), therefore we obtain from (15) that $H(\omega) \leqslant c_{9}|a|^{c_{10}}$, and

$$
H \leqslant c_{11}(1+\log |a|) \text {. }
$$

We have noted above that $D_{2} \neq 0$. But $D_{2}$ is the determinant of the system

$$
\begin{gathered}
x \alpha^{(k)}+y \beta^{(k)}+z \gamma^{(k)}=\lambda^{(k)}=\mu^{(k)} / \zeta^{(k)}, \\
x \bar{\alpha}^{(k)}+y \bar{\beta}^{(k)}+z \bar{\gamma}^{(k)}=\bar{\lambda}^{(k)}=\bar{\mu}^{(k)} / \bar{\zeta}^{(k)}, \\
x \bar{\alpha}^{(l)}+y \bar{\beta}^{(l)}+z \bar{\gamma}^{(l)}=\bar{\lambda}^{(l)}=\bar{\mu}^{(l)} / \bar{\zeta}^{(l)}
\end{gathered}
$$

$\dagger$ This is Theorem 2 from Фельдман (1971). The condition connected with the equation (46) of this work is taken away in Фелъдман (1972). 
and from (6) and (17) we obtain

$$
|x|,|y|,|z| \leqslant c_{12} \max _{1 \leqslant j \leqslant 2 n}\left|\mu^{(j)}\right| e^{c_{13} H} \leqslant c_{14}|a|^{1 / 2 n} e^{c_{11}(1+\log |a|)} \leqslant c_{1}|a|^{c_{2}} .
$$

THEOREM 2. Suppose that there are distinct indices $i, j$ such that the numbers (3) are linearly independent over $\mathbf{Q}$. Then all the solutions of (1) satisfy (4).

Proor. Let $a \neq 0$. If for any pair $i, j$ the numbers (3) are linearly independent over $\mathrm{Q}$ then the assertion of the theorem follows from Theorem 1 . Let $k, l, k \neq l$, be the indices for which the numbers (3) are linearly independent. Consider the nontrivial solutions in integers of the equation

$$
\Delta_{k l 1} X-\Delta_{k l 2} Y+\Delta_{k l 3} Z=0 .
$$

Let $x_{1}, y_{1}, z_{1}$ and $x_{2}, y_{2}, z_{2}$ be two such solutions. Without loss of generality we suppose that $\Delta_{k l 1}$ and $\Delta_{k l 2}$ are linearly independent over $Q$ and $z_{1} z_{2} \neq 0$. We have

$$
\left(x_{1} z_{2}-x_{2} z_{1}\right) \Delta_{k l 1}-\left(y_{1} z_{2}-y_{2} z_{1}\right) \Delta_{k l 2}=0
$$

and it implies

$$
x_{1} z_{2}-x_{2} z_{1}=y_{1} z_{2}-y_{2} z_{1}=0, \quad x_{1}=\left(z_{1} / z_{2}\right) x_{2}, \quad y_{1}=\left(z_{1} / z_{2}\right) y_{2}, \quad z_{1}=\left(z_{1} / z_{2}\right) z_{2},
$$

Thus all the nontrivial integer solutions of the equation (18) are proportional to each other. Let $x_{0}, y_{0}, z_{0}$ be the integer solution of the equation (18) with the minimal positive sum $x^{2}+y^{2}+z^{2}$; then all the other nontrivial integer solutions of the equation (18) are of the form

$$
x=t x_{0}, \quad y=t y_{0}, \quad z=t z_{0}, \quad t \in \mathbf{Z} .
$$

If the triplet $x, y, z$ satisfies the equation (1) then we have the equality

therefore $t^{2 n} \mid a$ and

$$
t^{2 n} F\left(x_{0}, y_{0}, z_{0}\right)=a,
$$

$$
|x| \leqslant\left|x_{0}\right||a|^{1 / 2 n}, \quad|y| \leqslant\left|y_{0}\right||a|^{1 / 2 n}, \quad|z| \leqslant\left|z_{0}\right||a|^{1 / 2 n} .
$$

As there exist only $C_{n}^{2}$ combinations of the indices $k, l$ all the solutions of (1) satisfying one of the equations (18) satisfy the inequality (4) with the corresponding $c_{1}$ and $c_{2}$.

For the solutions of (1) which do not satisfy any of the $C_{n}^{2}$ equations (18) the inequality (12) takes place. Therefore they satisfy the inequality (4).

THEOREM 3. Suppose that for any pair of distinct indices $i, j$, one of the numbers (3) is distinct from zero. If after substituting a solution from the equation

$$
x \Delta_{i j 1}-y \Delta_{i j 2}+z \Delta_{i j 3}=0
$$


(which has a nontrivial integer solution) in (1) the left-hand side of the obtained equation is not proportional to a power of a linear form or a power of a quadratic form with real coefficients then all the solutions of the equation (1) satisfy the inequality (4).

Proof. Let $x, y, z$ be the solution of the equation (1). If for these $x, y, z$ for any pair $i, j$ the equality (19) does not take place, then as we have already seen above (see the proof of Theorem 1) the inequality (4) takes place. Let for some pairs of different indices $k, l$ the equality

$$
x \Delta_{k l 1}-y \Delta_{k l 2}+z \Delta_{k l 3}=0
$$

take place. If for each of these pairs among the numbers $\Delta_{k l s}, s=1,2,3$, there are two linearly independent over $Q$ then the numbers $x, y, z$ satisfy the inequality (4) (see the proof of Theorem 2). Thus we must only consider the case when for some pairs $k, l$ among the numbers $\Delta_{k l s}, s=1,2,3$, any two of them are linearly dependent over $\mathbf{Q}$. By the hypothesis one of them is not zero. Let $\Delta_{k 13} \neq 0$. The triplet $x, y, z$ satisfies equations (1) and (20), therefore

$$
z=A x+B y, \quad A, B \in \mathbf{Q},
$$

and from (1) we get

$$
\prod_{i=1}^{2 n}\left(\left(\alpha^{(l)}+A\right) x+\left(\beta^{(t)}-B\right) y\right)=a .
$$

It is evident that $\alpha^{(t)}+A$ and $\beta^{(t)}+B \in \mathbf{K}^{(t)}$. Let $C, A_{1}=A C, B_{1}=B C \in \mathrm{Z}$; then the equation

$$
\prod_{t=1}^{2 n}\left(\left(C \alpha^{(t)}+A_{1}\right) x+\left(C \beta^{(t)}+B_{1}\right) y\right)=a C^{2 n}=a_{1}
$$

has rational integer coefficients. Let us expand the left-hand side to the irreducible factors. If among them there is a form of the third degree $f(x, y)$ then (4) holds by the theorem of the work of Фельдман (1971), as any $x, y$ satisfying equation (21) satisfy one of the finite set of equations

$$
f(x, y)=a_{2}, \quad a_{2} \in Z, \quad a_{2} \mid a_{1} .
$$

If among the irreducible factors there are only the forms of first and second degree then there are only four possible cases.

1. Among the irreducible factors there is a form

$$
\left.g(x, y)=p x^{2}+q x y+r y^{2}=p(x+(\rho+i \sigma) y)(x+\rho-i \sigma) y\right), \quad \rho, \sigma \in \mathbf{R}, \quad \sigma \neq 0 .
$$

Then the rational integer

$$
|g(x, y)|=|p|\left((x+\rho y)^{2}+\sigma^{2} y^{2}\right) \leqslant\left|a_{1}\right|=|a| C^{2 n},
$$

and thus $|y| \leqslant c_{11}|a|$, and $|x|$ and $|y|$ satisfy the inequality (4). 
2. Among the irreducible factors there are two non-proportional linear forms $k_{1} x+l_{1} y$ and $k_{2} x+l_{2} y$. Then for any solution of equation (21) we have

$$
k_{1} x+l_{1} y=a_{3}, \quad k_{2} x+l_{2} y=a_{4}, \quad a_{3}, a_{4} \in Z, \quad a_{3}\left|a_{1}, \quad a_{4}\right| a_{1},
$$

and again $|x|$ and $|y|$ satisfy the inequality (4).

3. Among the irreducible factors there are two non-proportional quadratic forms. Then

$$
p_{1} x^{2}+q_{1} x y+r_{1} y^{2}=a_{5}, \quad p_{2} x^{2}+q_{2} x y+r_{2} y^{2}=a_{6}, \quad a_{5}, a_{6} \in Z, \quad a_{5}\left|a_{1}, \quad a_{6}\right| a_{1} .
$$

Eliminating $x$ we obtain the equation

$$
\left|\begin{array}{cccc}
p_{1} & q_{1} y & r_{1} y^{2}-a_{5} & 0 \\
0 & p_{1} & q_{1} y & r_{1} y^{2}-a_{5} \\
p_{2} & q_{2} y & r_{2} y^{2}-a_{6} & 0 \\
0 & p_{2} & q_{2} y & r_{2} y^{2}-a_{6}
\end{array}\right|=0 .
$$

The left-hand side of this equation is diffierent from zero, its coefficients are rational integers, their moduli not greater than $c_{13}|a|^{c_{12}}$ and thus $|x|$ and $|y|$ satisfy (4).

4. Among the irreducible factors there are linear and quadratic forms. Then

$$
p_{3} x^{2}+q_{3} x y+r_{3} y^{2}=a_{7}, \quad k_{3} x+l_{3} y=a_{8}, \quad a_{7}, a_{8} \in Z, \quad a_{7}\left|a_{1}, \quad a_{8}\right| a_{1},
$$

and the estimate (4) is true again.

\section{References}

A. Baker (1968), 'Contributions to the theory of Diophantine equations, 1', Philos. Trans. Roy. Soc. London Ser. A, 263, 173-191.

Н. И. Фелъдман (1971), Эффективное степенное усиление теоремы Лиувилля Изв. AH CCCP, серия матем., 35, 973-990.

Н. И. Федъдман (1972), 'Одно неравенство, связанное с линейными формами от логарифмов алгебраических чисел', ДАН СССР, 207, 41-43.

W. Schmidt (1971), 'Linearformen mit algebraischen Koeffizienten, 2', Math. Ann. 191, 1-20.

A. Thue (1909), 'Über Annaherungswerte algebraischen Zahlen', J. reine angew. Math. 135, 284-305.

Department of Mathematics and Mechanics

Moscow State University

Moscow, 117234

U.S.S.R. 